\title{
LA-UR-20-23434
}

Approved for public release; distribution is unlimited.

Title: PAIN Schubert Review: Experiment

Author(s): $\quad$ Winch, Nicola M.

Madden, Amanda Christine

Marcath, Matthew James

Nelius, Eric Waggoner

Mayo, Douglas R.

Intended for: Shubert Review

Issued: $\quad 2020-05-07$ 
Disclaimer:

Los Alamos National Laboratory, an affirmative action/equal opportunity employer, is operated by Triad National Security, LLC for the National Nuclear Security Administration of U.S. Department of Energy under contract 89233218CNA000001. By approving this article, the publisher recognizes that the U.S. Government retains nonexclusive, royalty-free license to publish or reproduce the published form of this contribution, or to allow others to do so, for U.S. Government purposes. Los Alamos National Laboratory requests that the publisher identify this article as work performed under the auspices of the U.S. Department of Energy. Los Alamos National Laboratory strongly supports academic freedom and a researcher's right to publish; as an institution, however, the Laboratory does not endorse the viewpoint of a publication or guarantee its technical correctness. 


\section{PAIN Schubert Review: Experiment}

These slides detail the experimental section of the PAIN Schubert Review.

The PAIN project is investigating the utility of portable neutron radiography for emergency response.

Further sections give an overview of the PAIN project and detail the simulation performed. 


\section{Experimental Review Agenda}

- Introduction

- Detector Characterizations

- Neutron Generator Characterizations

- Scatter Characterization

- Betatron vs DT generator 


\section{Experimental goals}

- Test current state of the art performance

- Characterize detectors

- Flat panel

- Storage phosphor

- Characterize generators

- Flux

- Spot size

- Inform simulations

- Scatter

- Building system models

- Predict the future
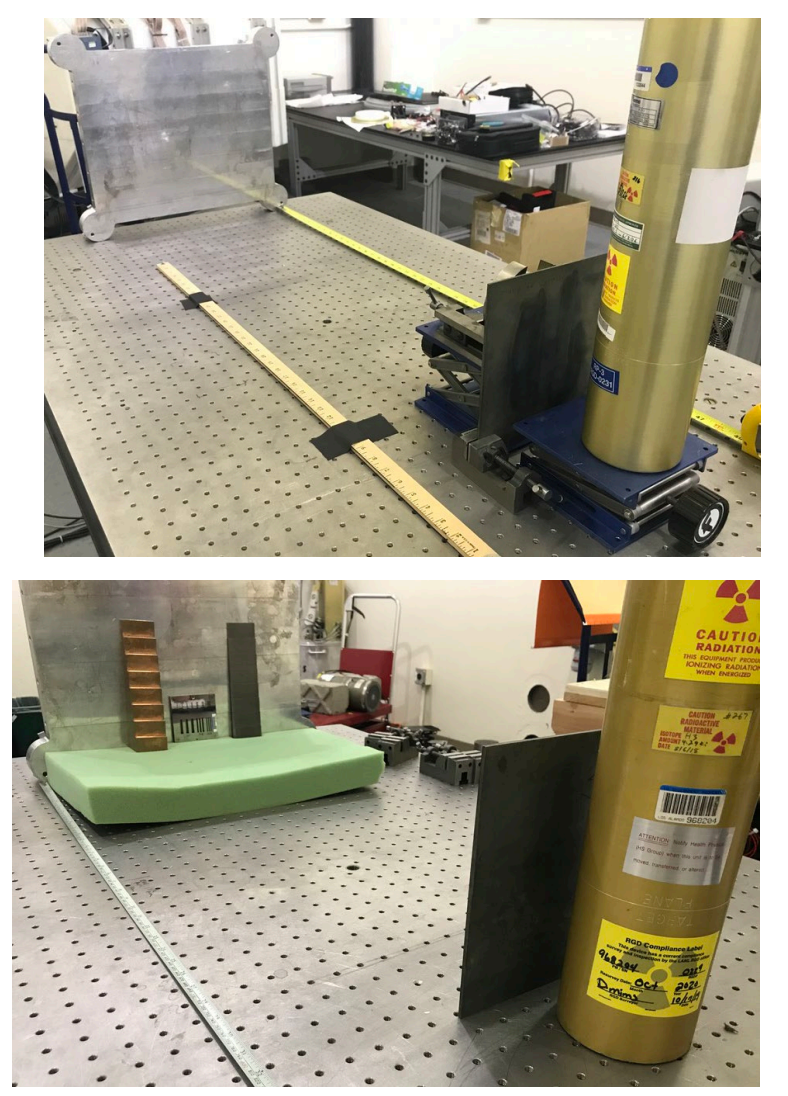

Experiment

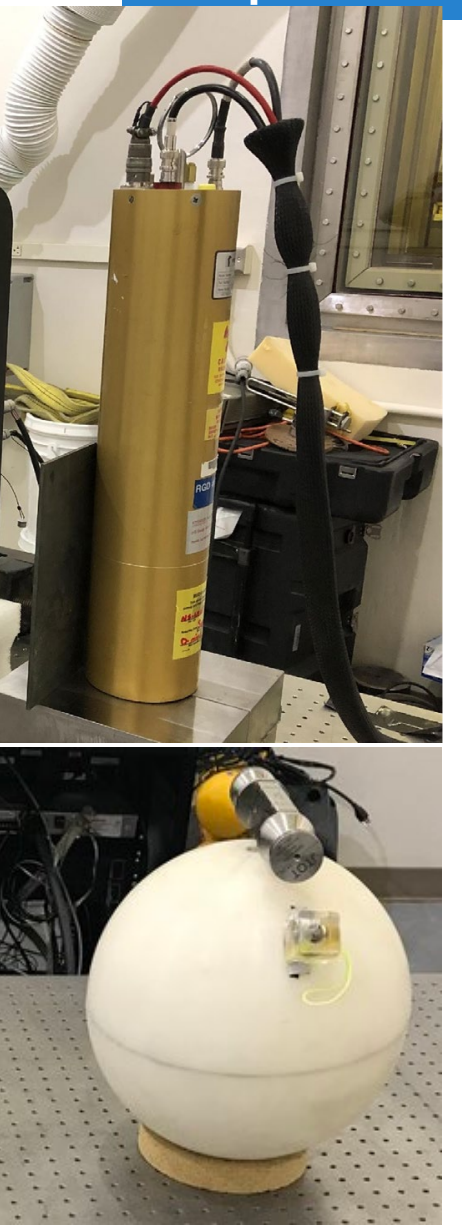




\section{Detector Characterization}

Experiment

Flat Panel Syء

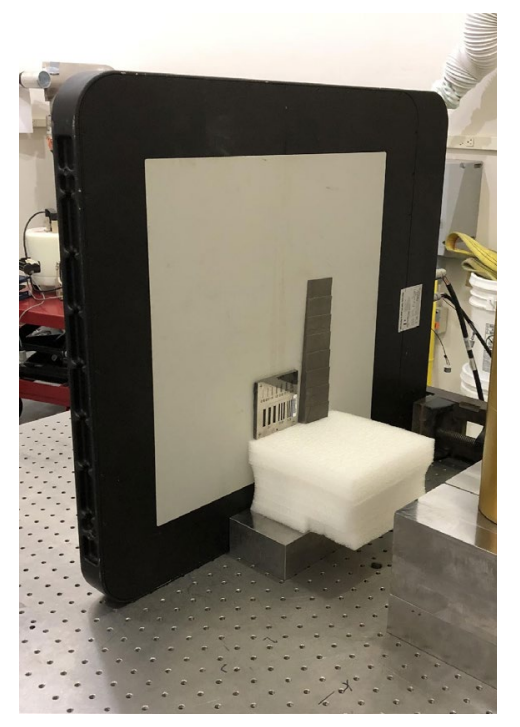

- Perkin Elmer 162

- $2.4 \mathrm{~mm}$ thick $\mathrm{Zn} s$ scintillator
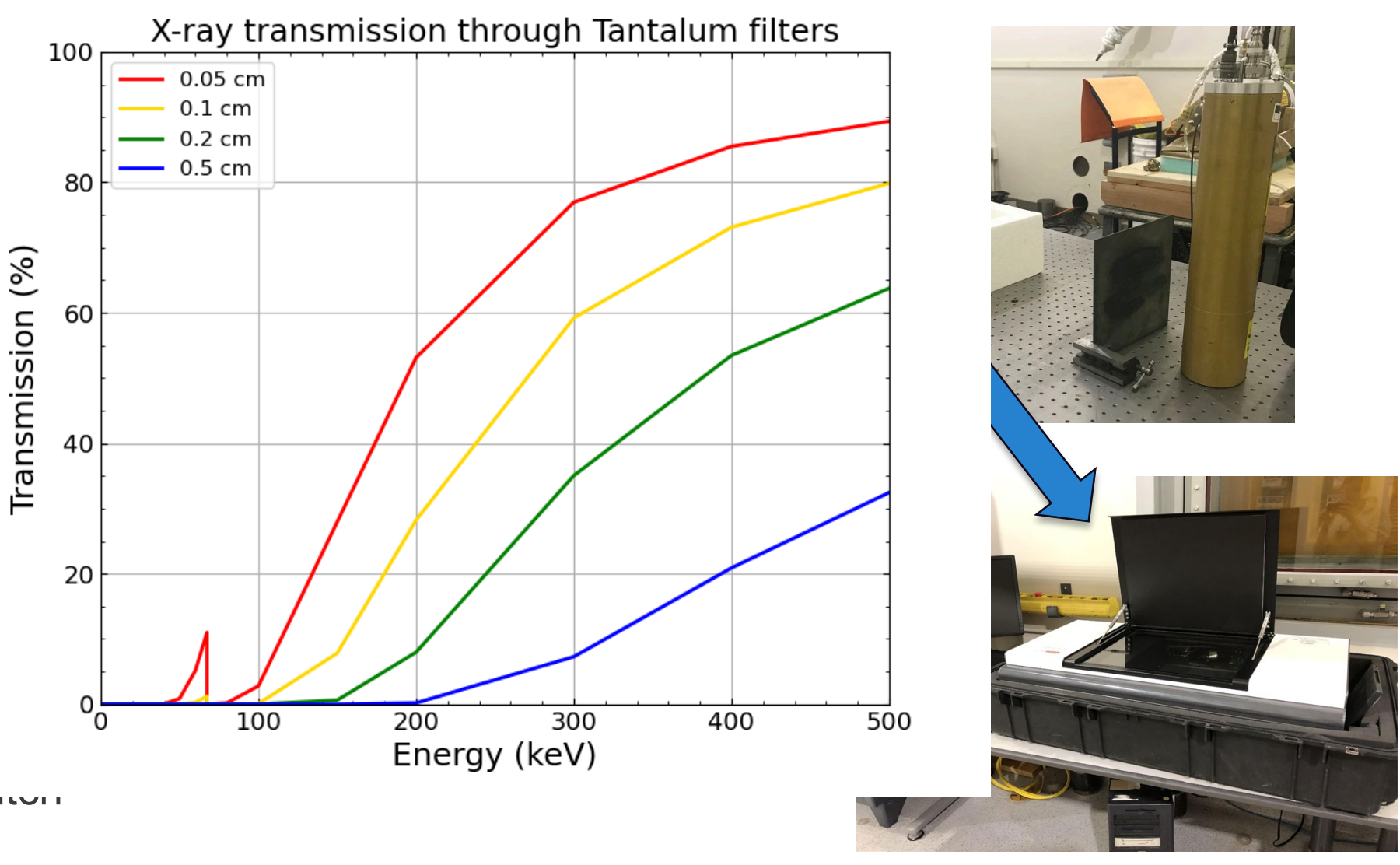

- 200 micron pixel 


\section{Flat Panel vs Storage Phosphor}

Experiment

Flat Panel

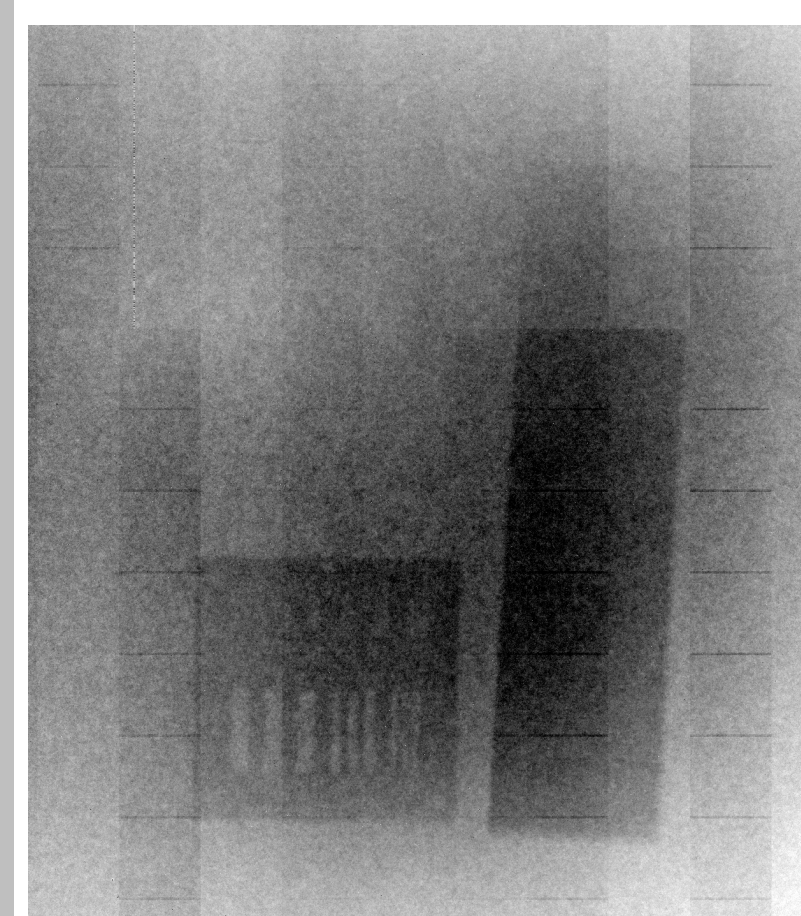

Storage phosphor (single) Storage phosphor (stack)

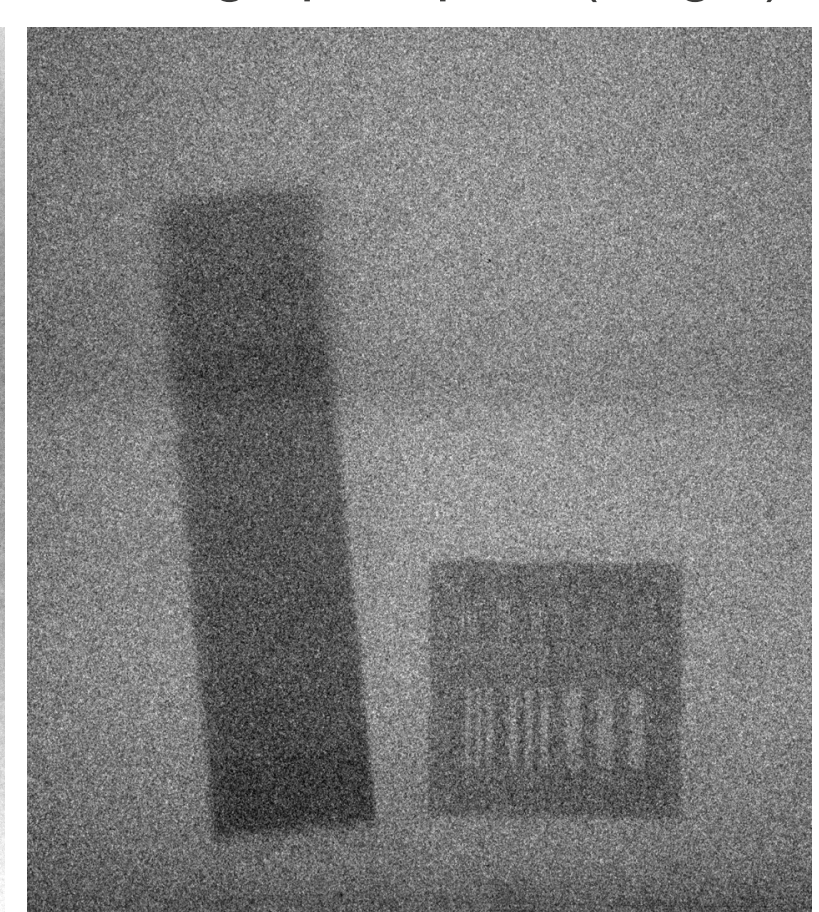




\section{Flat Panel vs Storage Phosphor}

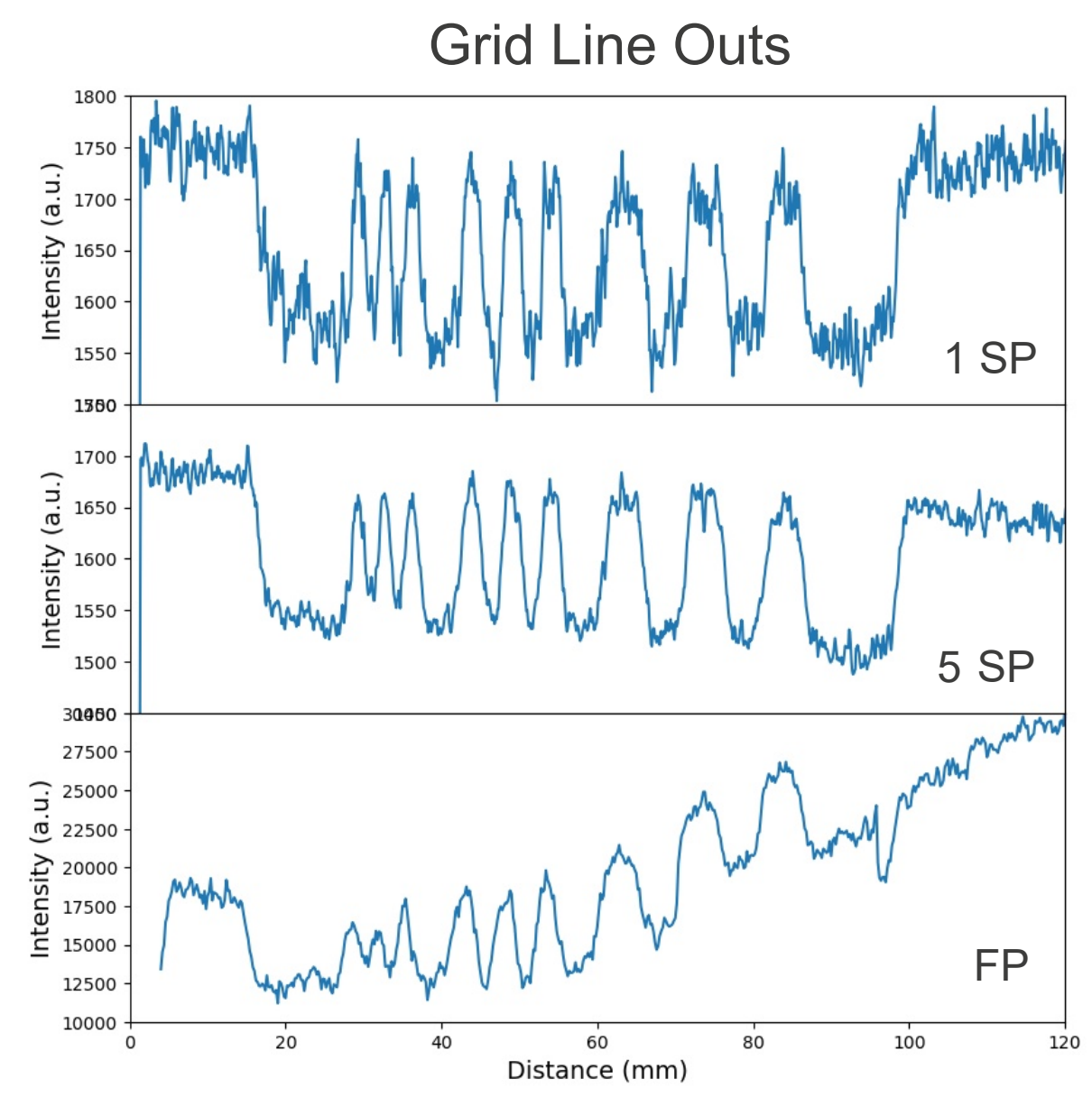

Step Wedge Line Outs

Experiment

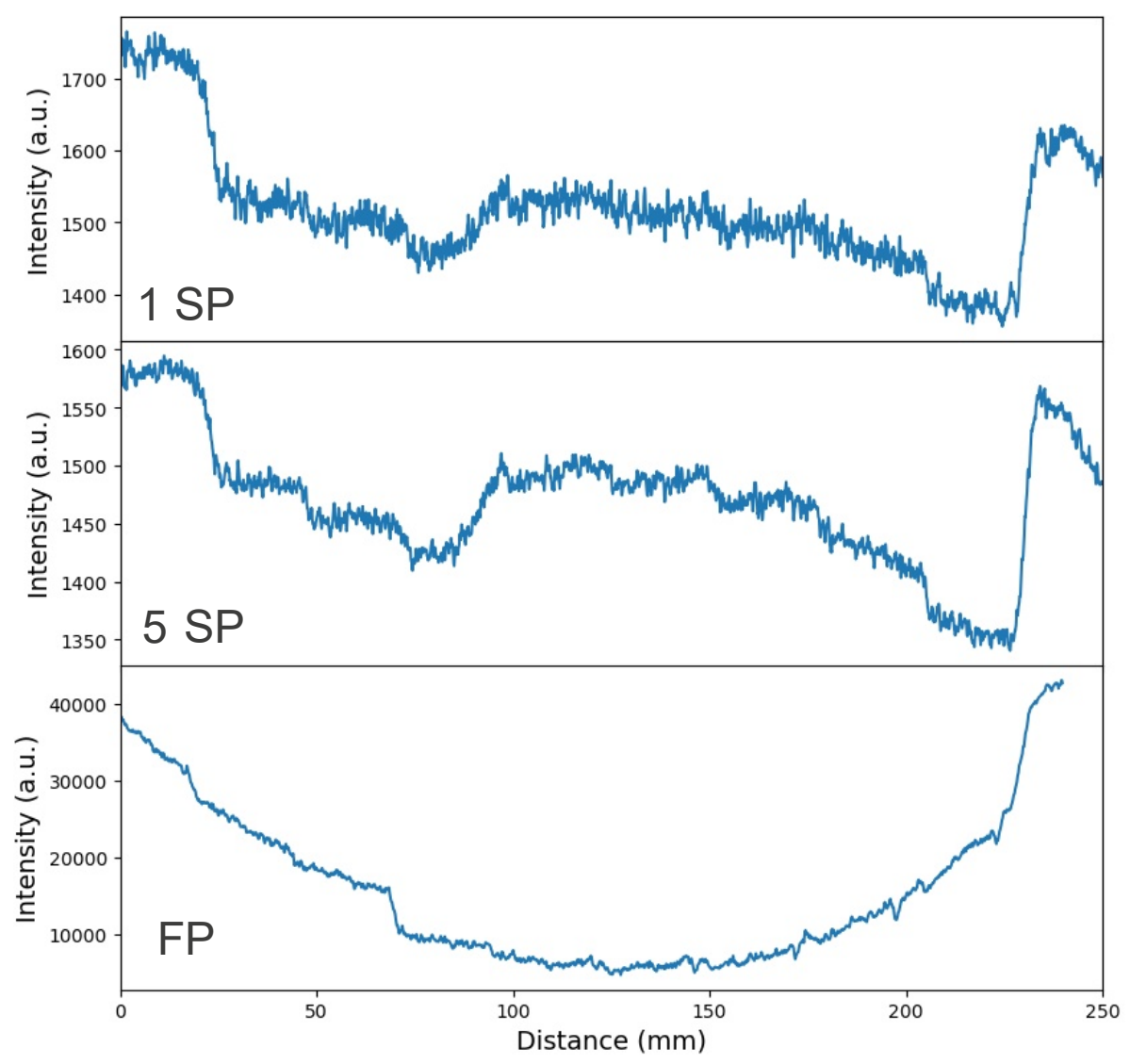




\section{Flat Panel vs Storage Phosphor}
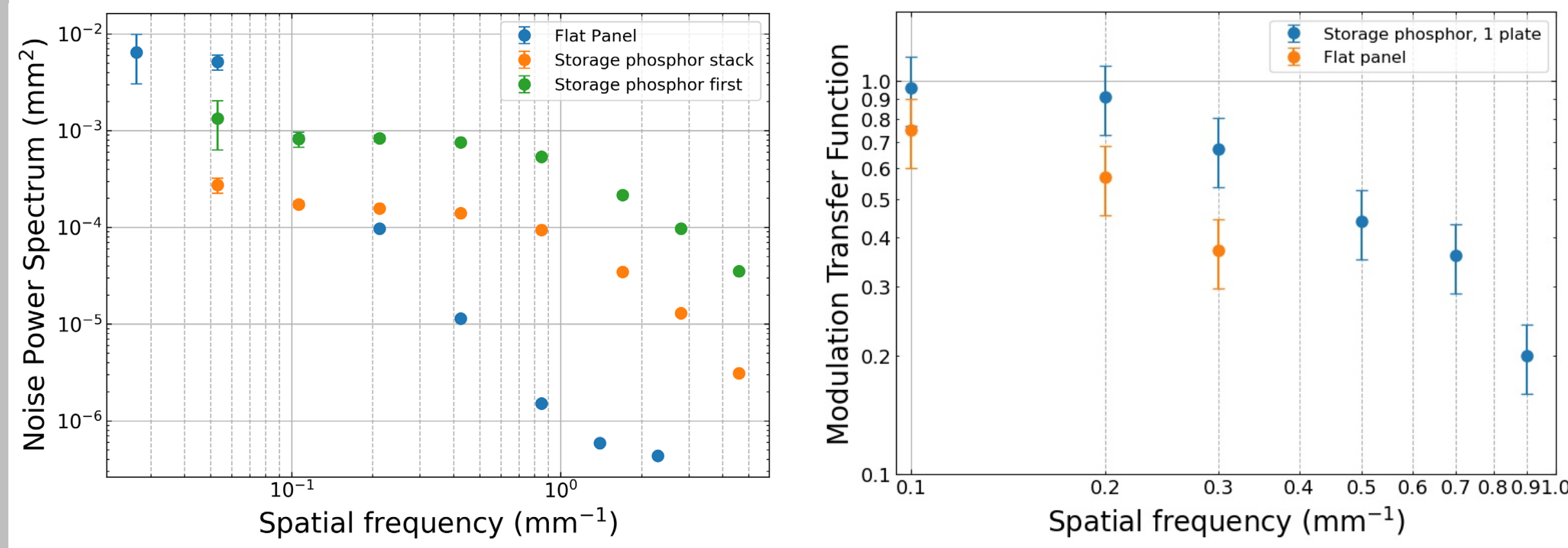


\section{Flat Panel vs Storage Phosphor}

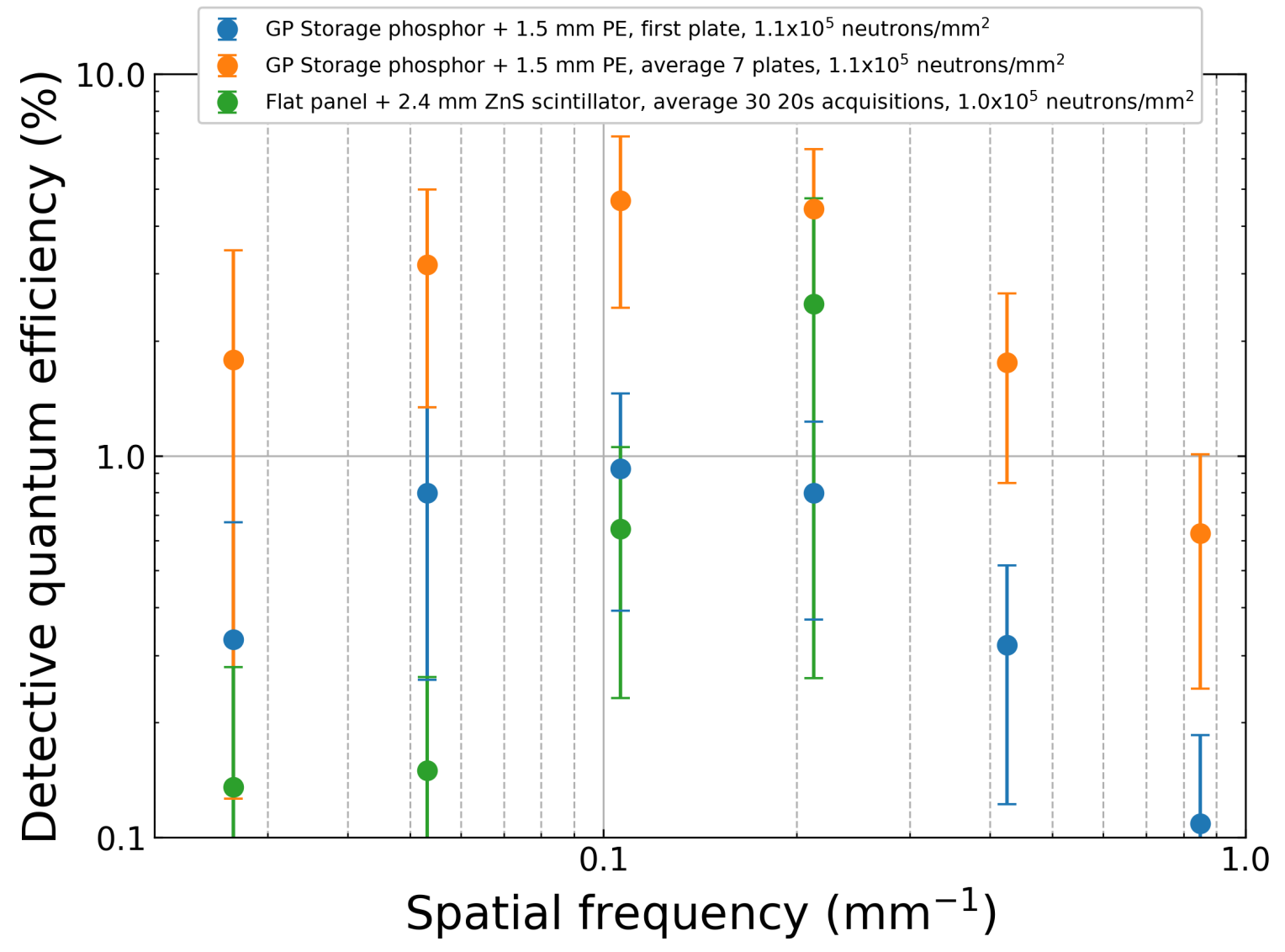




\section{Stacking storage phosphors}

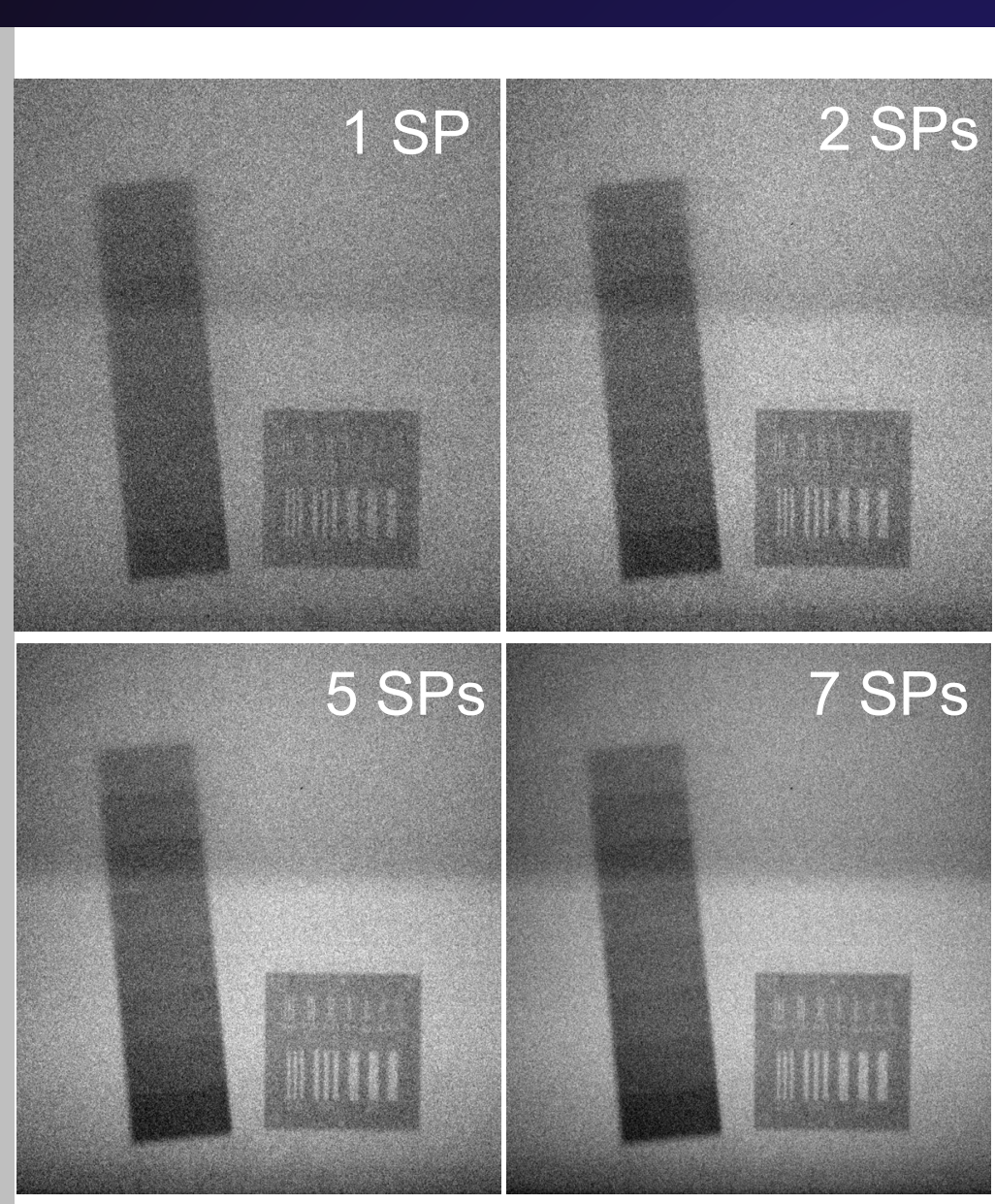

Experiment

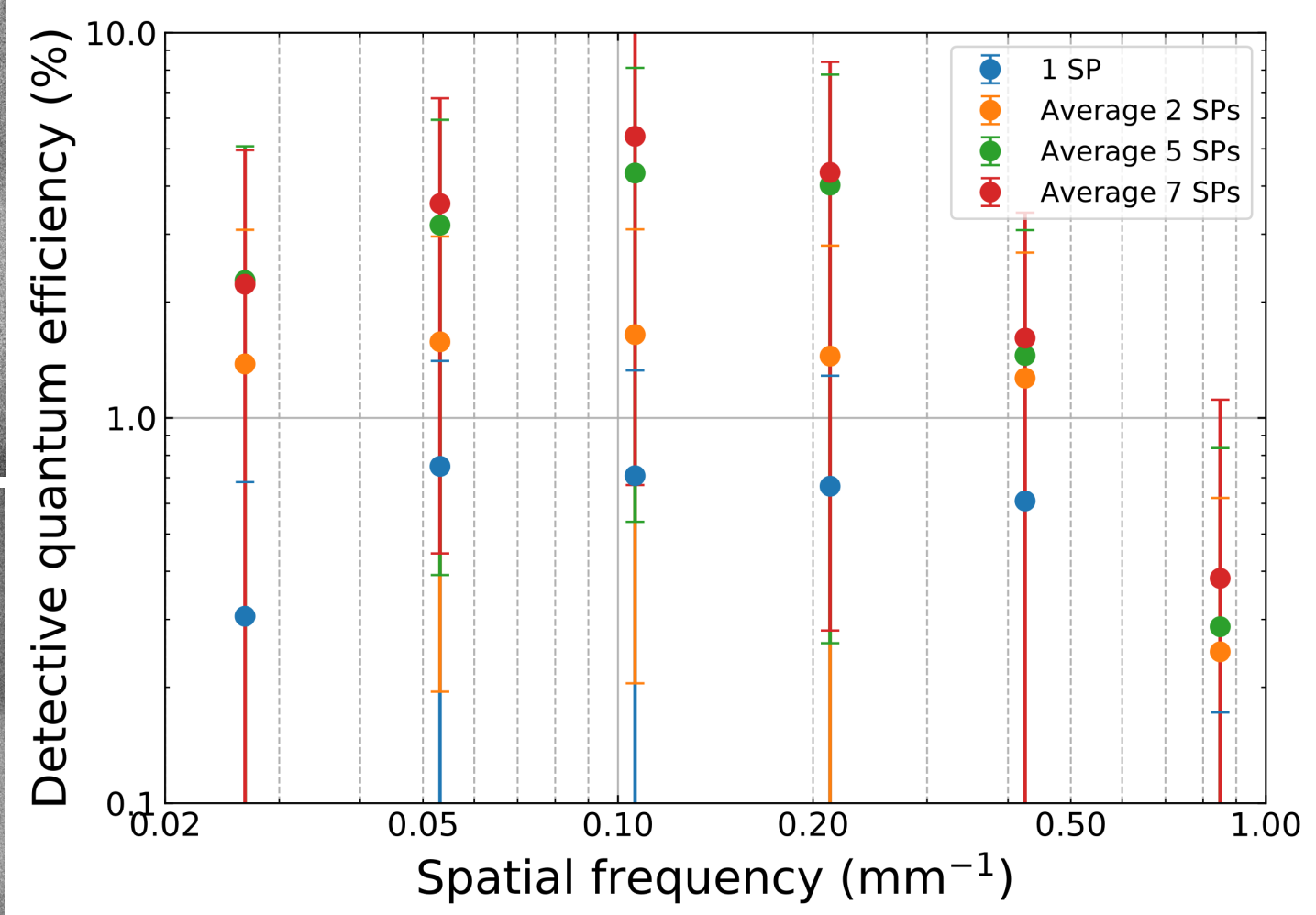

$1.5 \mathrm{~mm}$ thick PE 


\section{Thickness of converter material}

\section{7 layers}

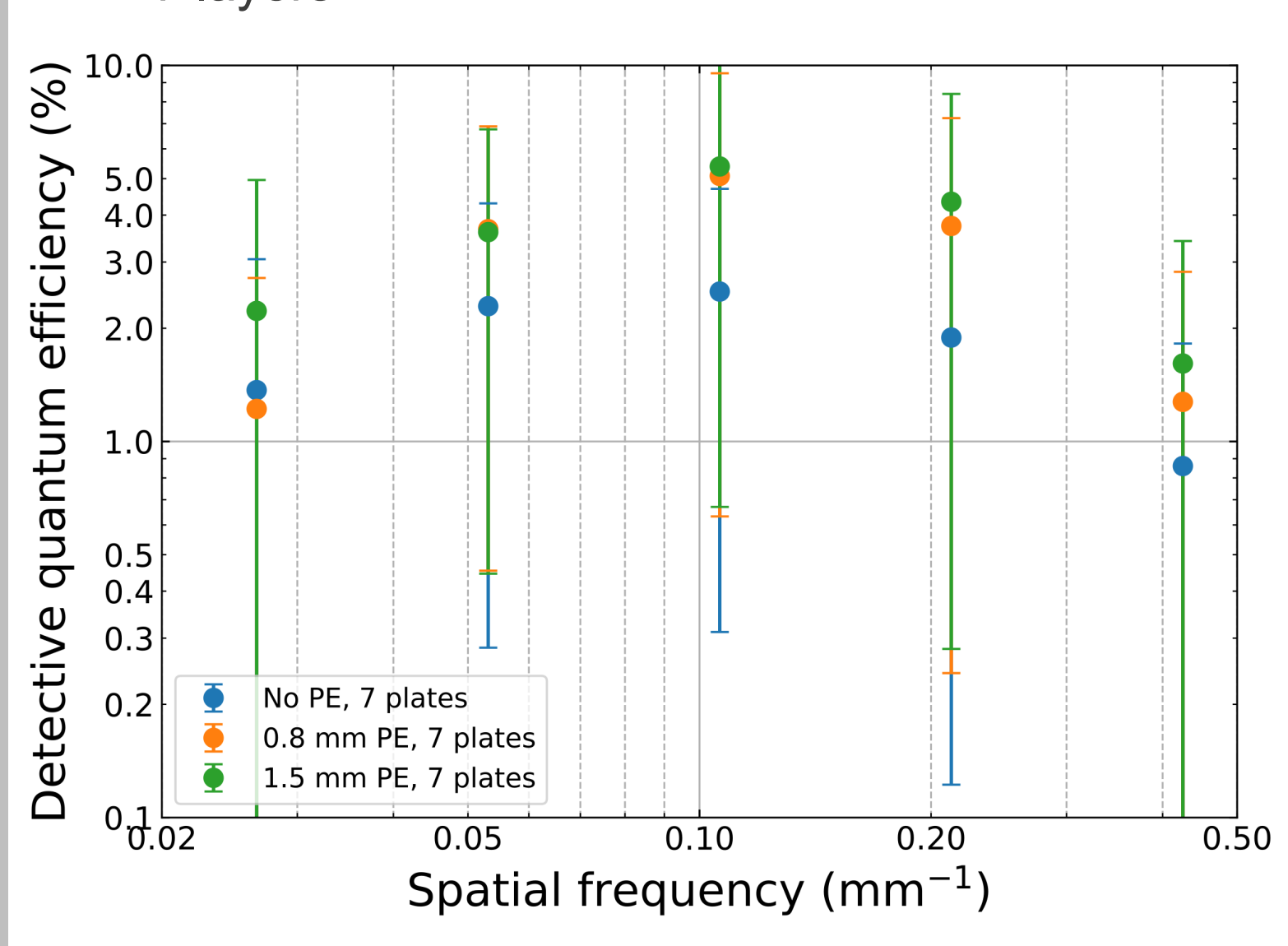

\section{No PE}




\section{Thermo Fisher Flux Measurements}

Experiment

- Radiation Effects and Reliabilities Team

- ISR Division

- Measured flux from Thermo Fisher neutron generators with multiple detectors

- Identified Albatross neutron detector as best measurement tool

- Gamma insensitive

- No dose limit

- Agreed within reason to manufacturer's specifications

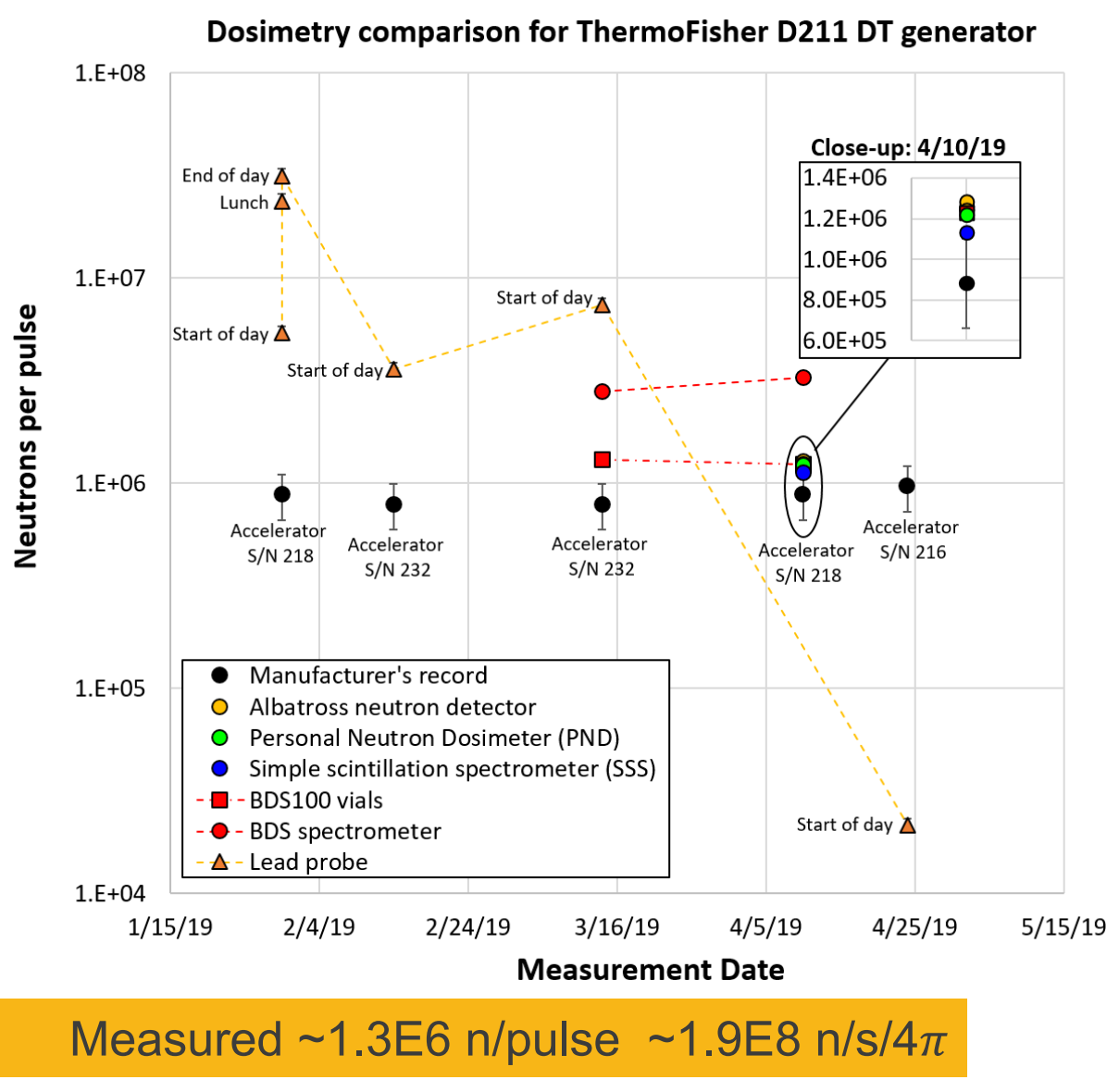




\section{Thermo Fisher Spot Size}

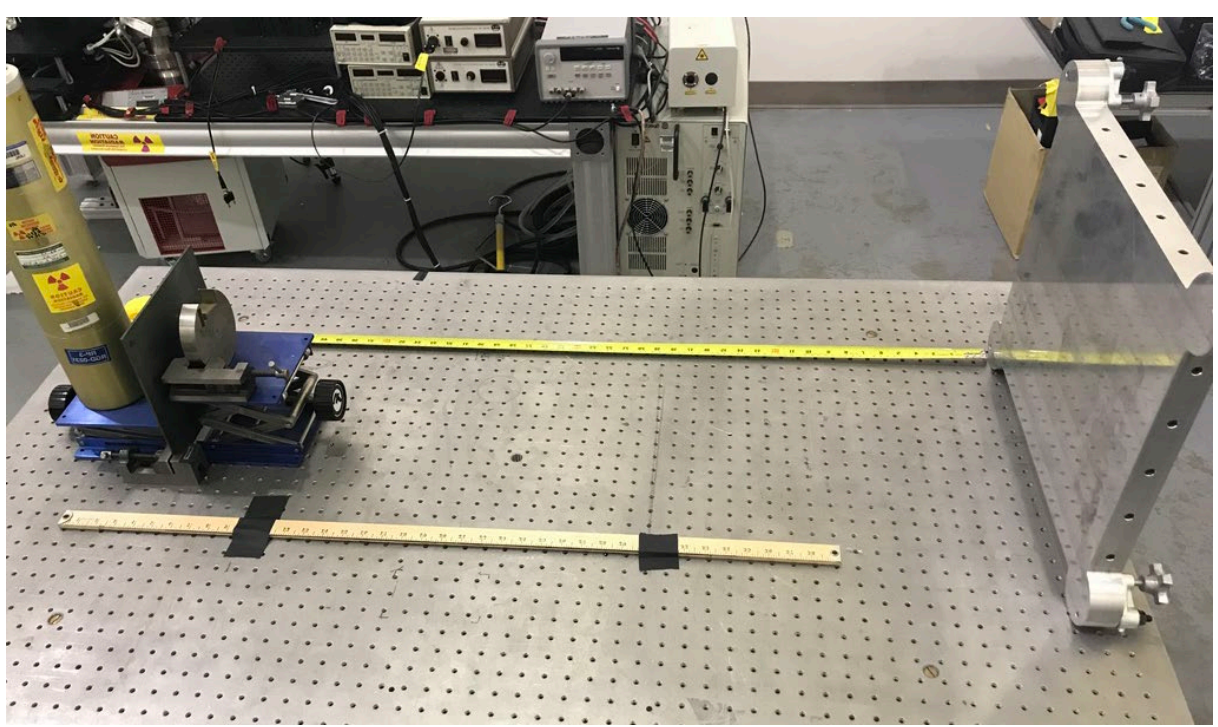

Experiment

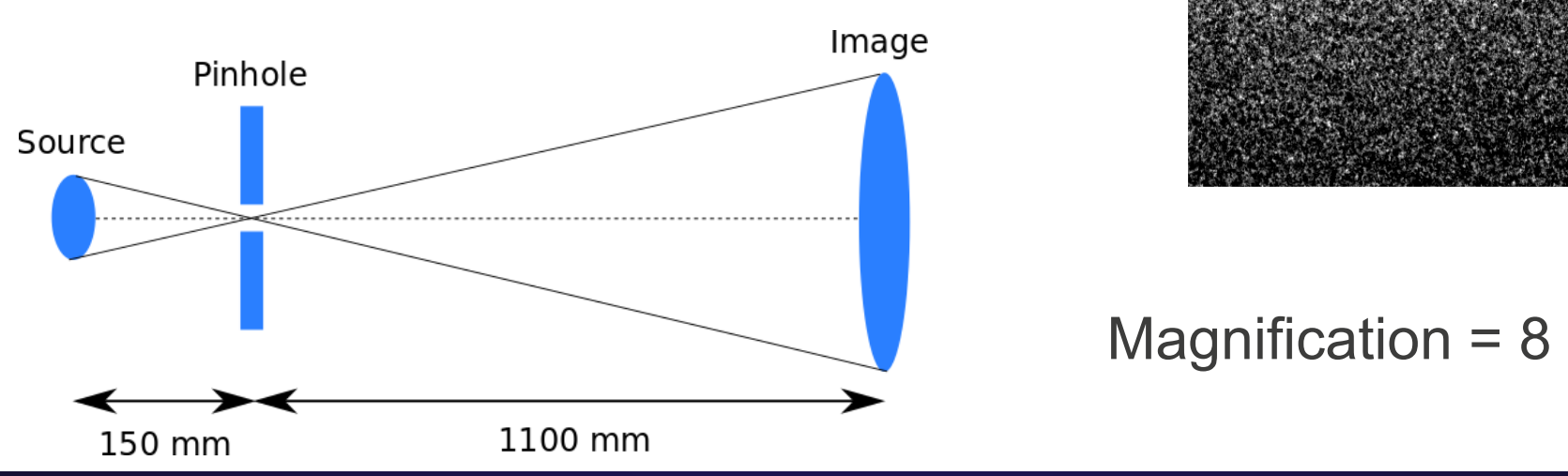




\section{Thermo Fisher Spot Size}
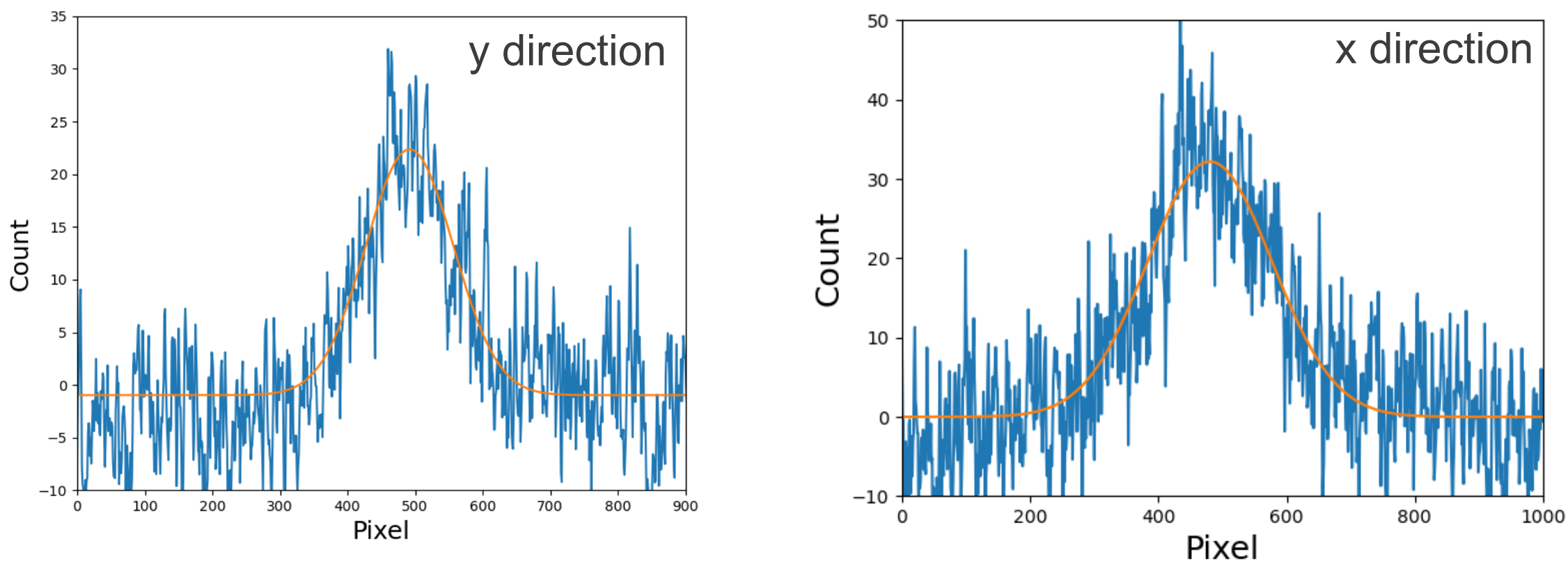

\begin{tabular}{|l|c|r|}
\hline Direction & $\mathrm{d}_{\text {FWHM }}(\mathrm{mm})$ & $\mathrm{d}_{\text {LANL }}(\mathrm{mm})$ \\
\hline $\mathrm{X}$ & $3+/-2$ & $5+/-3$ \\
\hline $\mathrm{Y}$ & $2+/-1$ & $3+/-3$
\end{tabular}




\section{Scatter characterization}

- Varying the object to detector position -At detector, 6" from detector, 12" from detector

- Fixed source to object distance of 36 "

- On table and on the floor
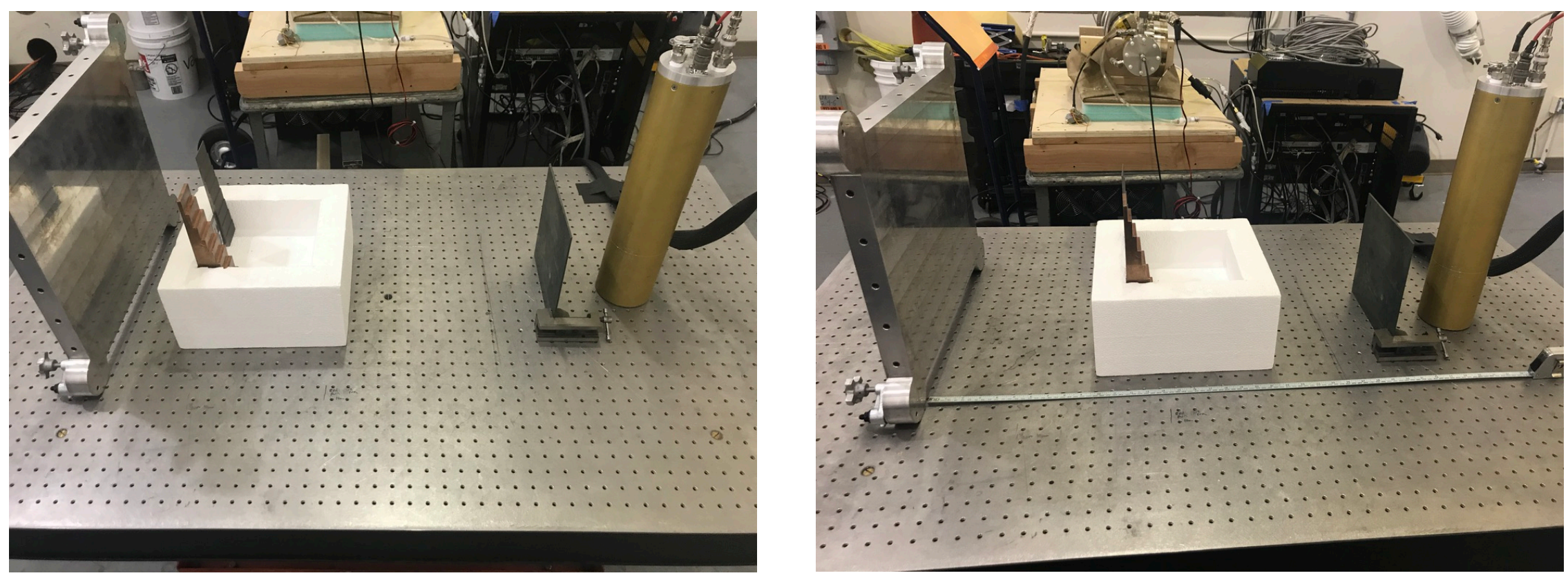


\section{Scatter Characterization}

Preliminary analysis will look at line outs of step wedge and resolution grid

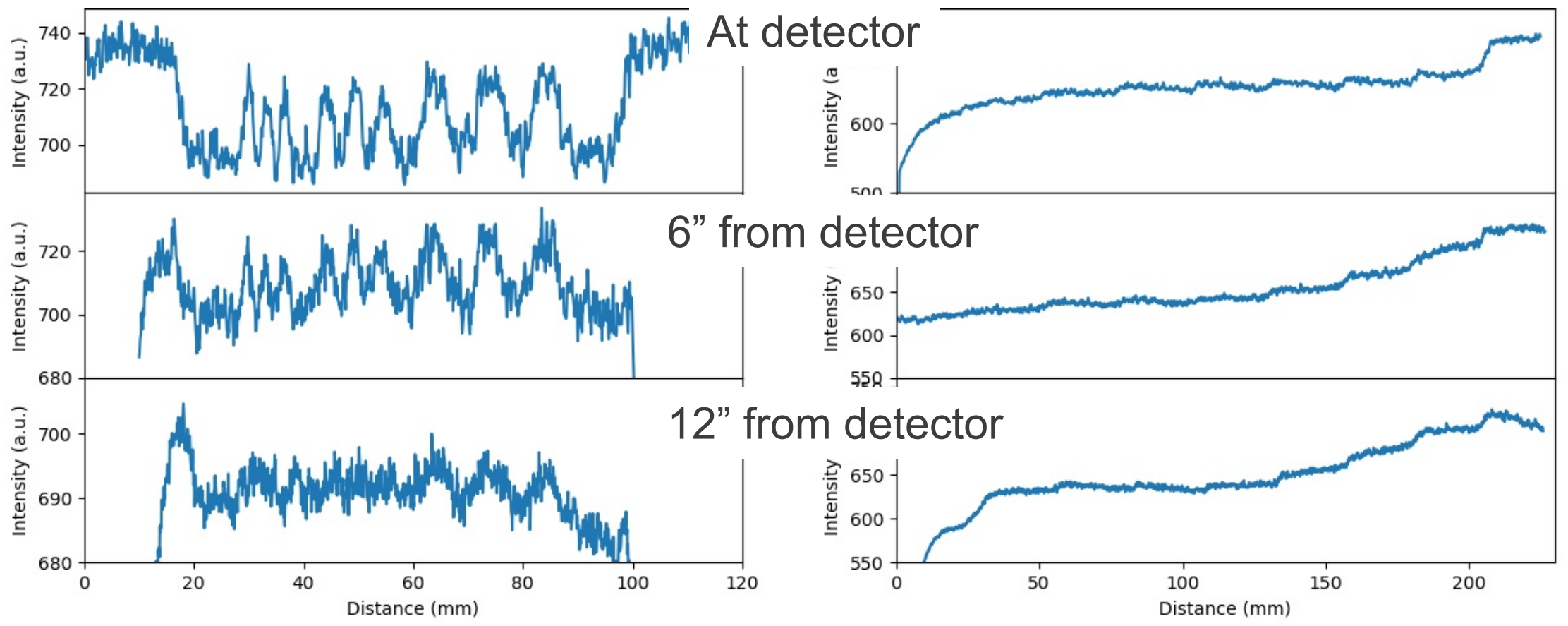




\section{Betatron vs DT generator}

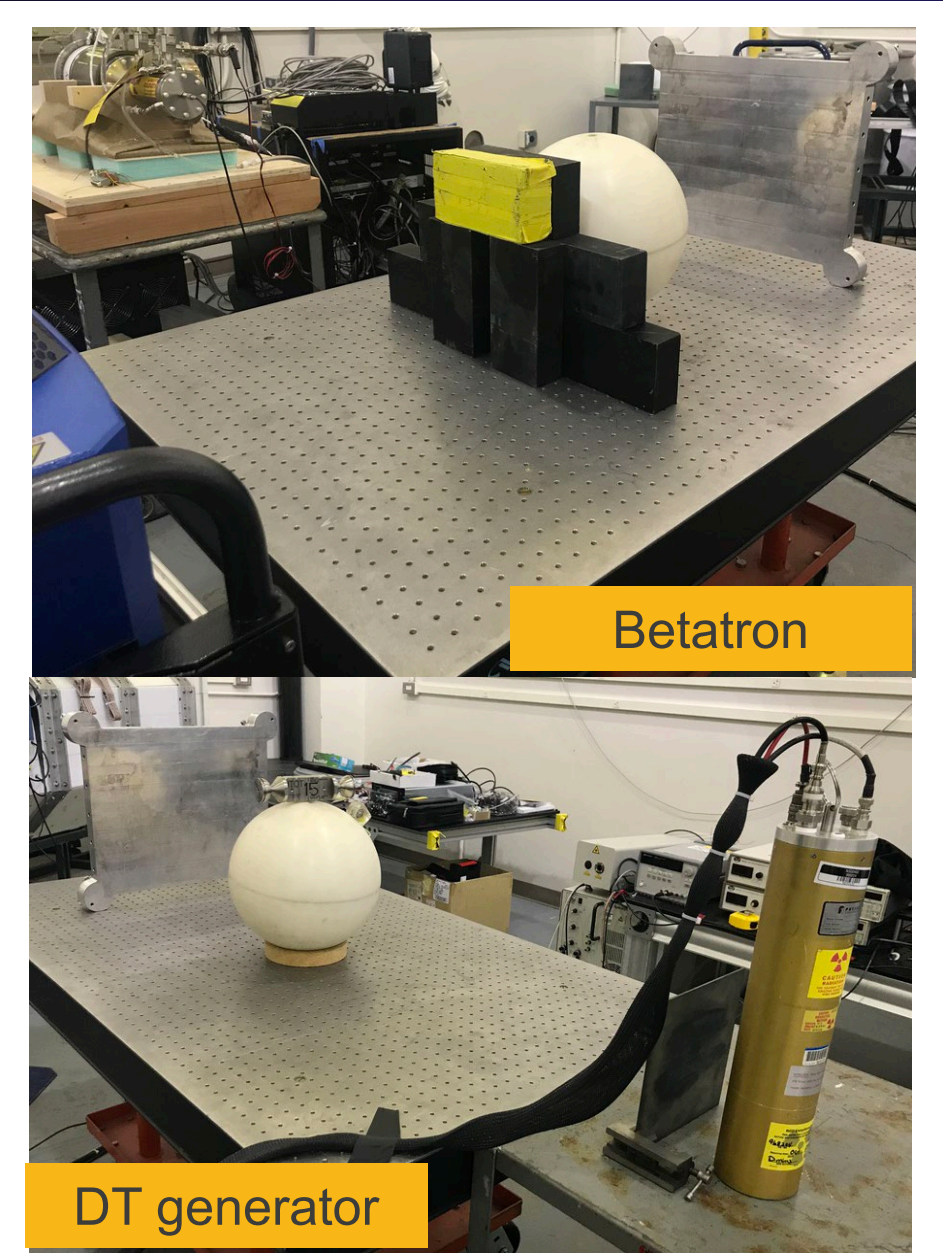

French Test Object

Experiment 


\section{Betatron vs DT generator}
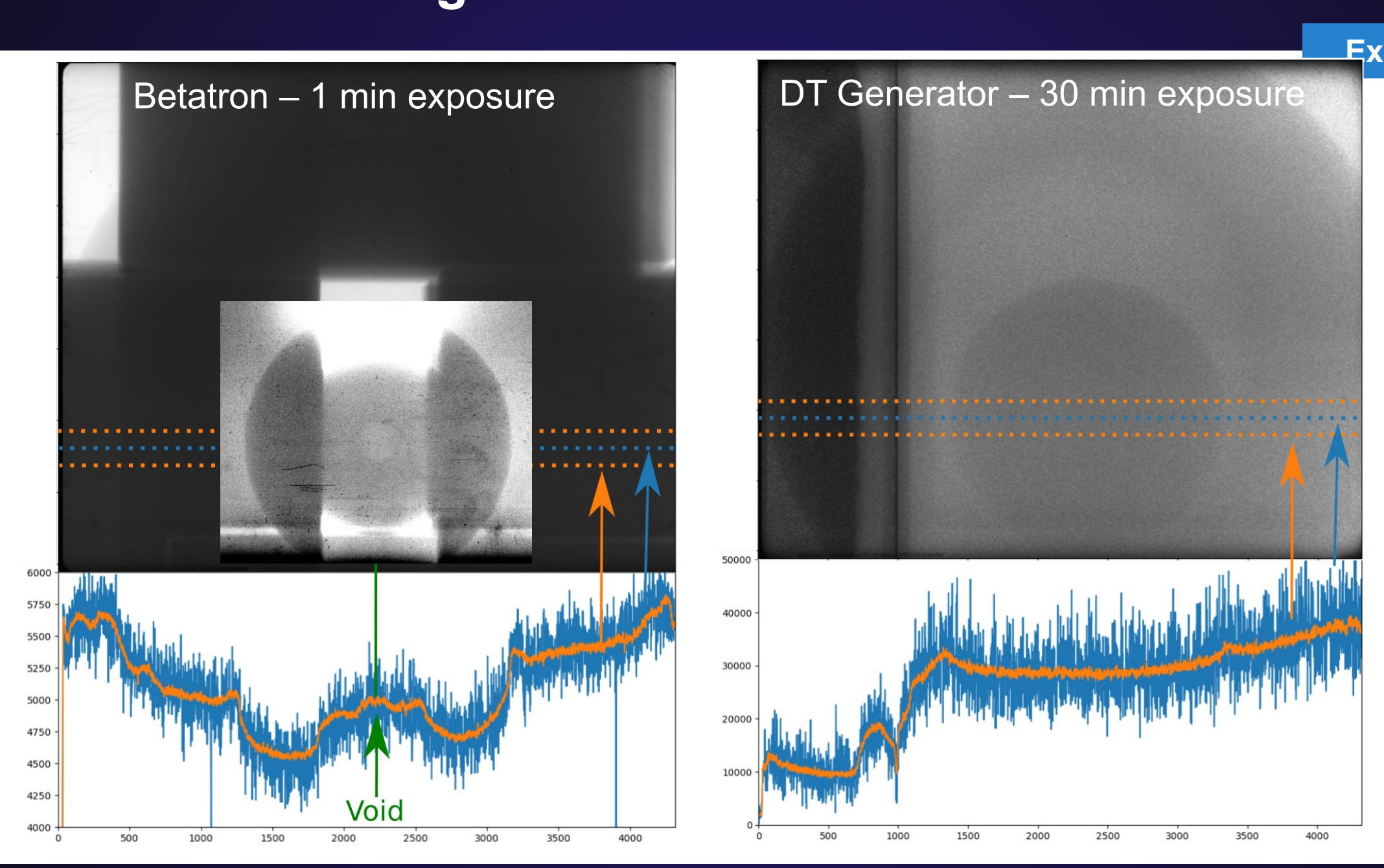